\title{
Aerodynamic and thermal interference of turbulent separated flows over building models
}

\author{
Aleksey Gnyria ${ }^{1}$, Sergey Korobkov ${ }^{1}$, Anton Koshin ${ }^{1, *}$, and Victor Terekhov ${ }^{2}$ \\ ${ }^{1}$ Tomsk State University of Architecture and Building, Russia \\ ${ }^{2}$ Kutateladze Institute of Thermal Physics SB RAS, Russia
}

\begin{abstract}
The paper presents experimental results of a physical simulation and computations of aerodynamic and thermal interference of turbulent flows over two building models. The experimental setup, conditions, and results are described, and the evaluation of obtained results is given in this paper. Conclusions are drawn on the basis of this research.
\end{abstract}

\section{Introduction}

Aerodynamic and thermal inteference of separated flows plays an important role in studying wind pressure and heat loss of buildings during their operation. The relevant literature shows a variety of approaches to experimental [1-3] and numerical [4] investigations of these phenomena. These works are mainly devoted to the aerodynamic behavior of airflows when facing a tandem of two square or rectangular prisms.

Although the literature in this field illustrates a wide range of information, the problem of aerodynamic and thermal inteference is still relevant due to mixed results, a complicated structure of separated flows and, as consequence, the impossibility of developing a correct model of turbulent transfer. Presently, the interference process and its effect on the airflow can be predicted only by virtue of systematic experimental research and construction of generalized curves under certain conditions.

\section{Experimental setup}

The experimental setup for aerodynamic tests includes a wind channel with a process chamber of $0.42 \times 0.42 \mathrm{~m}$ size and $1.5 \mathrm{~m}$ length, experimental building models, and measuring devices. The schematic view of the arrangement of building models and the airflow visualization are presented in Fig. 1.

On the basis of the previous study [5], this work continues the investigations of aerodynamics and heat exchange between two square prisms arranged at different distances from each other $(L / b=0 \div 6)$ and the lateral shift $(D / b=1 \div 3)$, where $b$ is the prism side equaling $50 \mathrm{~mm}$ (Fig. 1).

\footnotetext{
${ }^{*}$ Corresponding author: dawghood@mail.ru
} 



Fig. 1. Schematic view of bulding models arrangement and oil flow visualization.

During the experiment, the distribution of pressure and heat transfer coefficients is measured on each side of the square prism. Oil flow visualization is performed on the bottom of the wind channel as shown in Fig. 1. A detailed description of the experimental technique is presented in [5]. The Reynolds number in this experiment varies between $2 \cdot 10^{4}-4 \cdot 10^{4}$.

\section{Results and discussions}

As can be seen from Fig. 1, the vortex structure is observed in the aerodynamic trail. In front of Model 1, the airflow separates and forms a horseshoe vortex. Then it flows over Model 2 and forms another horseshoe vortex, becoming asymmetric at the same time.

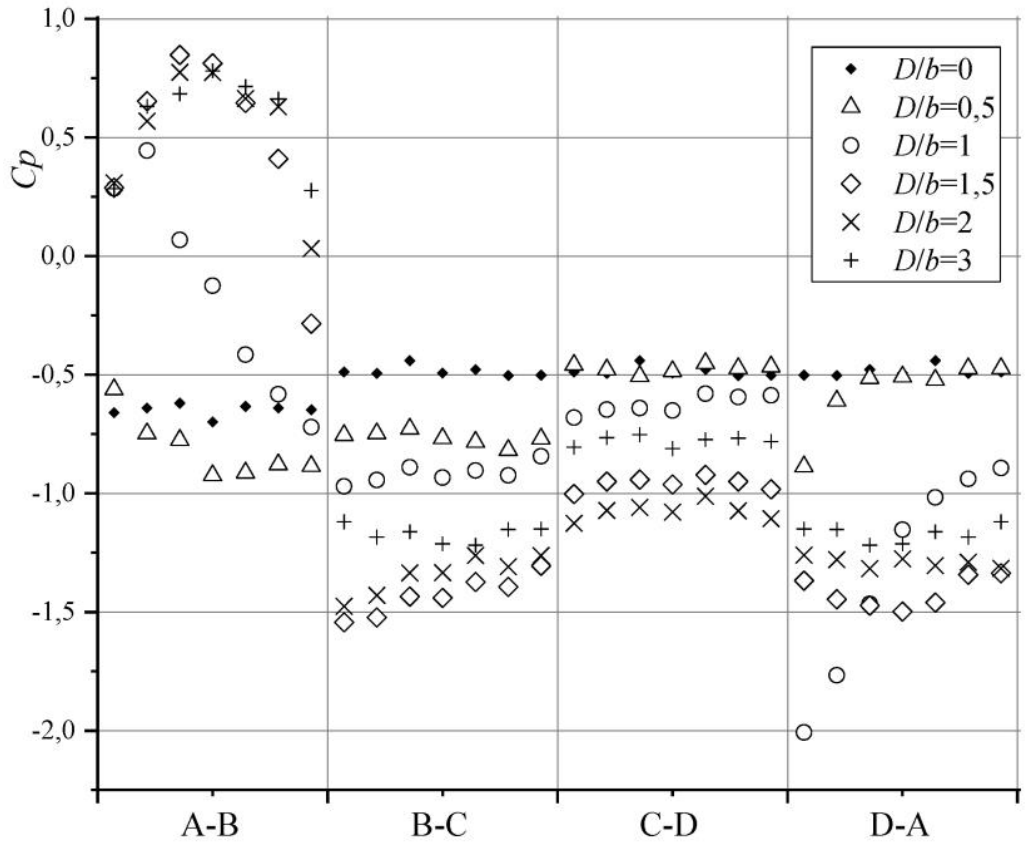

Fig 2. Distribution of pressure coefficients over sides of Model 2.

Measurement results on the pressure distribution demonstrate substantial differences in the wind pressure in Models 1 and 2. As Model 2 is positioned in the aerodynamic trail, it is subjected to a lesser pressure than Model 1. Moreover, the differential pressure occurs on $B-C$ and $D-A$ sides resulting in the lateral pressure on Model 2. The similar phenomena are 
observed during changes in heat transfer. Along $D-A$ side, the intensity of heat transfer from the surface of Model 2 is higher than that along $B-C$ side.

The coefficients of aerodynamic and thermal interference $I F$ can be found in the following way [1-3]. The coefficient of aerodynamic interference $I F_{C p}$ can be obtained from the ratio between the mean and the local values of the pressure coefficient $C p$ :

$$
I F_{C p}=\frac{C p_{\text {mean }}}{C p_{\text {mean }(o)}}
$$

The coefficient of thermal interference $I F_{N u}$ can be obtained from the ratio between the mean and the local value of the Nusselt number [1-3]:

$$
I F_{N u}=\frac{\overline{N u}_{\text {mean }}}{\overline{N u}_{\text {mean }(o)}}
$$

The dependence between the parameter and the lateral shift $D / b$ is presented in Fig. 3 . The value of $I F$ parameter is obtained via the minimum wind pressure which depends on $D / b$ ratio at different distances $L / b$ between square prisms (Fig. 1).



Fig. 3. Dependences between $I F$ parameter and lateral shift $D / b$.

According to Fig. 3, the aerodynamic interference $I F_{C p}$ achieves larger values at lower lateral shift of square prisms. This indicates the growth in air rarefaction on the leeward prism, affected by the separated flow. At the same time, the heat transfer changes not so intensively and $I F_{N u}$ coefficient is close to unity in each case.

From the outcome of our investigation it is possible to conclude that the aerodynamic and thermal interference is subjected to the following impacts. First, $A-D$ side when entering the airflow, enables the formation of differential pressure on the lateral sides of Model 2, thereby increasing the heat transfer on $D-A$ side. Second, the separated flow behind Model 1, forms accelerated airstreams which scatter as early as at $L / b=1.5$ and have a lower effect on the pressure coefficients and the Nusselt number at longer distances. Thermal interference turns to be more stable relative to the model arrangement.

This work was financially supported by Grant N 8780.2016.8 from the President of the Russian Federation. 


\section{References}

1. X.F. Yu, Z.N. Xie, J.B. Zhu, J. Wind Eng. Indust. Aerod. 142, 188 (2015)

2. Y. Hui, A.Yoshida, Y. Tamura, J. Fluids Structures 37120 (2013)

3. Y. Hui, A.Yoshida, Y. Tamura, J. Wind Eng. Indust. Aerod. 104-106, 98 (2012)

4. A. Lankadasu, S. Vengadesan, Int. J. Numerical Methods Fluids 571005 (2008)

5. A.A. Koshin, V.I. Terekhov, Conf. Energy Efficiency of Residential Buildings, 71 (2015) 